\title{
Models in Ecosystem Science
}

\author{
Charles D. Canham, Jonathan J. Cole, and \\ William K. Lauenroth
}

\section{The Role of Modeling in Ecosystem Science}

Quantitative models play an important role in all of the sciences. Models can range from simple regression equations and analytical models to complex numerical simulations. Their roles can vary from exploration and problem formulation to sophisticated predictions upon which management decisions are based. In the most basic sense, models express the logical consequences of a set of hypotheses and generate predictions (in the strictest sense) that can be compared with observations in the quest to falsify those hypotheses. Beyond this, the definitions and utility of models become controversial, and further discussion of models usually sparks an often intense debate over a host of both practical and philosophical issues. The ninth Cary Conference, held May 1-3, 2001, at the Institute of Ecosystem Studies, was designed to explore those debates, and to evaluate the current status and role of modeling in ecosystem science.

Beyond their fundamental use in testing hypotheses, models serve a number of functions in our quest to understand ecosystems. Quantitative models allow the investigator to observe patterns embedded in the data, to synthesize data on disparate components into an integrated view of ecosystem function, and ultimately to predict the future behavior of some aspects of the ecosystem under given scenarios of future external drivers (Figure 1.1). While the participants of Cary Conference IX found broad consensus for these uses of quantitative models, the conference also revealed strongly held preferences for different approaches to modeling. One of the major axes of contention, for example, was the tension between favoring simple or parsimonious models (Chapters 4 and 8 ) versus models that were more mechanistically rich (Chapter 5). Under the surface of this usually jovial disagreement between modelers of different schools lie deep philosophical differences about the nature of scientific understanding itself. In Chapter 2, Oreskes, the lone philosopher at the conference has articulated some of the relationships between science, philosophy, and modeling. 


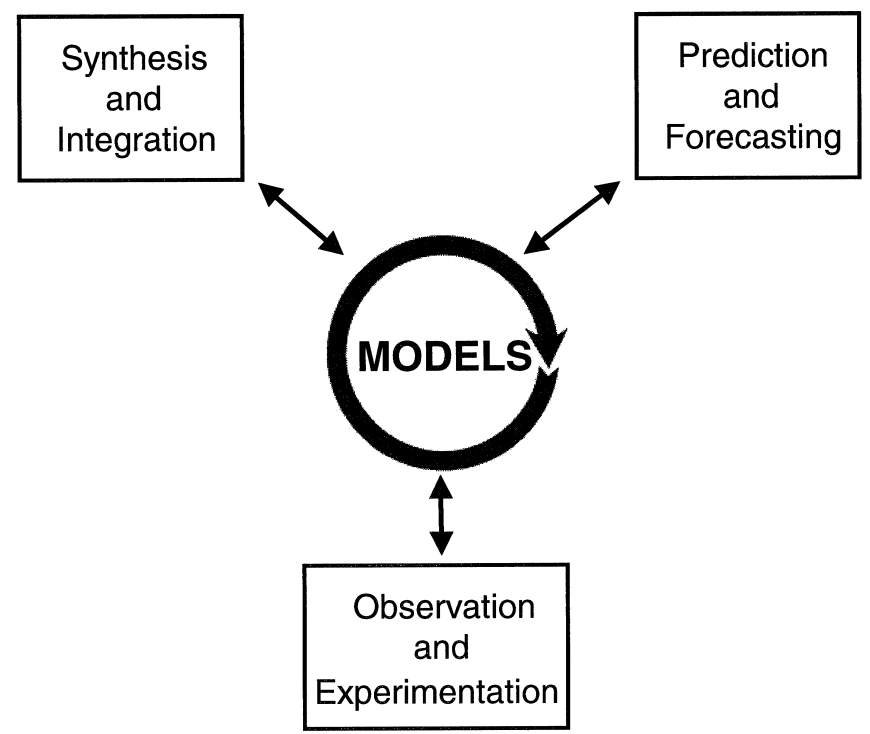

Figure 1.1

For the purposes of the Conference, we highlighted the roles of models in three distinct components of ecosystem science: observation and experimentation; synthesis and integration; and prediction and forecasting (Figure 1.1).

\section{Observation and Experimentation}

There are many examples in which models have provided the motivation for intensive empirical research. The most noteworthy is undoubtedly the "missing carbon sink" in the global carbon balance, although the carbon is only "missing" in the context of our models and/or our measurements. The mass-balance principles that led to the focus on the missing sink represent an important and useful constraint on ecosystem models. Pastor (Chapter 15) provides a powerful example of the use of mass-balance principles to suggest new field experiments for the study of plant competition.

Nonetheless, it is relatively rare to see tight integration between development of models in ecosystem science and the field research needed to generate the parameter estimates for the models. Modeling texts are replete with examples of failures when modeling is brought in as an afterthought (e.g., Starfield et al. 1990). There are many reasons for the lack of integration, including the generally weak modeling skills of many ecosystem scientists (Chapters 3 and 23). Ecosystem experiments are frequently designed to test fairly narrow hypotheses that do not require a formal model. In general, classical experiments answer 
qualitative hypotheses (Does treatment X have any effect on Y?). Quantitative models, on the other hand, require parameterization of the functional relationship between $\mathrm{X}$ and $\mathrm{Y}$. This is often better accomplished through comparative studies and regression than through the much more widely taught (and respected) analysis of variance and formal experimental design. Thus, experiments are particularly valuable in deciding what to include in a model but poorly suited to generate the functional relationships needed by the model.

\section{Synthesis and Integration}

There is a strong tradition of holism in ecosystem science, but it could be argued that much of the current research in the field focuses on new mechanisms and processes (boxes and arrows) as elaborations on traditional and simpler models. Ecologists can be justly accused of reveling in the complexity of nature, and ecology is frequently touted as the science of connections, but it is obvious that not all connections are equally important in governing any particular ecosystem state or process. Quantitative models can play an important role in helping us determine the most important processes and components for any given question (Chapter 6). Sensitivity analyses and the related techniques of path analyses and structural equation modeling (e.g., Grace and Pugesek 1997) can be used in the traditional sense of identifying parameter values that deserve rigorous attention in order to reduce model uncertainty, but they also serve a much broader role in helping us understand the strength of individual processes within the complex web of potential interactions that occur in ecosystems (Gardner et al. 1981; Fennel et al. 2001).

In a survey of attitudes about modeling, members of the Ecological Society of America (Chapter 3) were asked the most important reason for their use of models. The two most frequent responses were (1) to clarify conceptualization of system structure and (2) to clarify quantitative relationships between and among system components ( $>40 \%$ of respondents, combined). The use of models as an important tool for prediction was the third most common response, given by only $15 \%$ of respondents. While it is easy to focus on the details of the quantitative output of models, and many of the chapters of this volume address the quantitative evaluation of models (e.g., Chapters 8 and 13), many of them stress the more critical role of models in synthesizing our understanding of ecosystems (e.g., Chapters 4, 5, and 6) and in the teaching of ecology (e.g., Chapters 22 and 23).

\section{Prediction and Forecasting}

There was a great deal of discussion at the conference of the nature and limitations of prediction in ecosystem science. Beyond the narrow, scientific use of models to test hypotheses, ecosystem models are frequently used in public policy and natural resource management (Clark et al. 2001). There is considerable debate over our success at predicting the temporal dynamics of ecosystems (Chapter 2), and even over the philosophical validity of such predictions 
(Oreskes et al. 1994). Within the narrow confines of science, all model output can be defined as a prediction (see below), but as Bugmann points out in Chapter 21, there is real danger that the public perceives far more certainty in model predictions than is warranted. Pielke (Chapter 7) argues that conventional approaches to modeling are poorly suited to simultaneously meet scientific and decision-making needs.

There is considerable variation in the terminology used to describe model output, both within the chapters in this volume and within the field as a whole. As Pielke (Chapter 7) and Bugmann (Chapter 21) point out, the problem is compounded by differences between the narrow, scientific use of a term and the range of meaning imparted by the same term in a public arena. We considered but rejected the idea of trying to standardize terminology either before the conference or in this volume and, instead, present an attempt at a lexicon of modeling terminology later in this chapter.

\section{The Status of Modeling in Ecosystem Science}

Some divisions remain, but there appears to be broad acceptance of the important role of models in ecosystem science (Chapter 3 ). In contrast, a relatively small proportion of the papers in premier ecological journals have employed quantitative models (Chapter 3). Duarte et al. (Chapter 24) outline a number of impediments to both the development and the achievements of models. The impediments to model development are more prosaic, and technological advances constantly lessen the barriers through development of modeling software and availability of computing power. The impediments to the achievements of models are more fundamental and include limits to both prediction and understanding (Chapters 24 and 26).

Despite widespread acceptance of the value of models, modeling skills remain elusive. "Lack of training" was the most often cited limitation on the use of modeling by the respondents of the survey conducted by Lauenroth et al. (Chapter 3). One of the discussion groups at the conference focused on strategies to increase modeling skills among ecologists and identified a number of specific types of modeling skills that need to be developed, as well as specific suggestions for addressing those needs (Chapter 23). A second group considered the role of modeling in undergraduate education (Chapter 22). Their framework for improving the use of models in undergraduate ecology education is based on the premise that undergraduates at all levels would benefit from more explicit training in modeling.

A number of the chapters address another major limitation on ecosystem models: the availability of critical data for both model parameterization and model testing (e.g., Chapters 3,12, 13, and 27). This is, in part, a reflection of insufficient integration of modeling and empirical research (i.e., a disconnect between the needs of models and the objectives of field researchers). It also 
reflects the time and expense of collecting the necessary data, particularly for models that span large space and time scales.

\section{Simplicity versus Complexity in Ecosystem Models}

Models should be made as simple as possible, but not simpler.-adapted from a quote about theories attributed to Albert Einstein

All ecological models are, by definition, simplifications of nature (Chapter 2). Oreskes et al. (1994) argue that there is little empirical evidence that the world is actually simple or that simple accounts are more likely than complex ones to be true. They suggest that predilections for simplicity are largely an inheritance of seventeenth century theology. While Ockham's razor was originally sharpened for theological arguments and may not necessarily be the path to a full understanding of nature, there are many compelling reasons to keep models as simple as possible (e.g., Chapters 2 and 5).

Ecologists appear to differ widely in their predilection for simplicity and abstraction. These differences are apparent in the chapters in this volume (e.g., Chapters 4 and 5). We feel that the differences are healthy and that a diversity of approaches to modeling is as important as a diversity of approaches to science. How simple a model should be is part art form, part personal preference, but it is always determined by the nature of the question (Chapter 6). Moreover, our standards for what constitutes a "simple" model are likely to evolve as both our modeling abilities and our detailed understanding of natural phenomena evolve. Armstrong (Chapter 14) presents the arguments for the need to incorporate the size structure of organisms in ocean ecosystem models, particularly in the context of global carbon questions. Pastor (Chapter 15) provides an example in which very traditional competition models that ignore ecosystem science can be recast in the light of simple mass-balance principles. DeAngelis and Mooij (Chapter 5) argue for the benefits of "mechanistically rich" models. One of the benefits of such models is the rich array of outputs generated by the model. This variety allows comparison of diverse outputs against empirical data, providing more means to evaluate to model and develop confidence in the model (Chapter 24). A related limitation of such models is that associated with this rich array of outputs is a large amount of uncertainty (Chapter 8).

\section{A Selective Lexicon for Evaluating Ecosystem Models}

There are two ways of constructing a model: One way is to make it so simple that there are obviously no deficiencies, and the other way is to make it so complicated that there are no obvious deficiencies. The first method is far 
more difficult.—adapted from a quote by computer scientist C.A.R. Hoare on the subject of software design

A significant portion of the conference was devoted to the issue of evaluating ecosystem models. It became clear early in the conference that there was considerable difference of opinion not only over approaches to model evaluation, but also over the terminology used in this important effort. Conscious efforts to standardize terminology are almost always futile in science. In lieu of that, we present here a selective lexicon of the major terms and common usages expressed at the conference. We focus on two areas: model testing and the nature of model output.

\section{Model Testing}

Validation. As Gardner and Urban point out in Chapter 10, the process of model validation has "been surrounded with an inordinate degree of confusion." The on-line Merriam-Webster Dictionary (www.m-w.com) defines "validate" as "to support or corroborate on a sound or authoritative basis." Oreskes et al. (1994, 642) echoed this definition by arguing that validation of models "does not necessarily denote an establishment of truth.... Rather, it denotes establishment of legitimacy." Thus, "a model that does not contain known or detectable flaws and is internally consistent can be said to be valid" (Oreskes et al. 1994, 642). As they point out, the term is commonly used in a much broader (and to their minds, inappropriate) sense as a general determination that the model provides an accurate representation of nature. Hilborn and Mangel (1997), in a monograph on "confronting models with data," don't include the term "validation" in their index, although there is some discussion of the issue in a section on distinguishing between models and hypotheses. As they point out, there is a common view that models should be "validated" through comparisons between model predictions and data. However, all models will disagree with some of the data. Thus, "models are not validated; alternate models are options with different degrees of belief" (Hilborn and Mangel 1997, 31; see under "Confirmation," below). Burnham and Anderson (1998) provided a detailed summary of the statistical methods for evaluation of alternate models, using the principles of maximum likelihood and information theory.

The usage advocated by Oreskes et al. (1994) (and implicitly by Hilborn and Mangel 1997) focuses on model structure rather than on model output. This is a subtle but important distinction. As Rastetter points out in Chapter 12, evaluation of alternate model structures can present much greater challenges than determination of the goodness of fit of any particular model structure to a set of data (Chapter 8). While alternate model formulations may not differ significantly in their fit to a particular data set, they may invoke vastly different mechanisms, with important consequences when the model is used in novel conditions (Chapters 12 and 13). Burke et al. (Chapter 13) provide an example of this through an analysis of the implications of seemingly minor differences in the equations used to characterize the temperature dependence of decomposi- 
tion in biogeochemical models. The process of evaluating model structure is clearly critical enough to warrant a specific term, and "validation" appears to be the best candidate.

Calibration. As Oreskes et al. (1994) pointed out, we frequently have better data on ecosystem responses (the dependent variables) than on the processes that drive those responses (the independent variables). They define "calibration" as "the manipulation of the independent variables to obtain a match between the observed and simulated" dependent variables. Aber et al. (Chapter 11) note that most large ecosystem simulation models are calibrated, in the sense that free parameters (unconstrained by actual measurements) have been adjusted to make the model output match the observed data (or to simply produce reasonable patterns). Aber et al. considered this a weakness of those models.

Regression is a form of calibration in which rigorous statistical procedures can be used to determine the values of parameters that optimize the fit between observed data and the predictions of the regression model. The principles of maximum likelihood (Hilborn and Mangel 1997), information theory (Burnham and Anderson 1998), and Bayesian statistics (Chapter 9) extend the familiar concepts of regression to provide a very powerful framework for rigorous parameter estimation and testing of alternate models. These principles are often used in the development of component submodels within large ecosystem simulation models, but they are also eminently suitable for the simpler statistical models presented by Håkanson in Chapter 8 .

Confirmation. There is a natural temptation to claim that a match between observed data and predicted model results "confirms" the model. As Oreskes et al. (1994) pointed out, this is a logical fallacy ("affirming the consequent"). The concordance could be a result of chance rather than of the verity of the model. In contrast, if the match is poor, the model can logically be called flawed in some way. On the other hand, scientists consider hypotheses that are not refuted by repeated comparisons to data to gradually gain "confirmation." The bottom line is that we can never truly verify a model, just as we can never fully "prove" a hypothesis. We can, however, develop various degrees of confidence in models.

Adequacy and Reliability. Gardner and Urban (Chapter 10) suggest replacing the term "validation" with more specific terms that measure the utility and explanatory power of a model. "Adequacy" is the degree to which a model "explains" the observed set of ecological dynamics. "Reliability" is the degree to which model behaviors or predictions are within the observed set of ecosystem behaviors. They present a formal method (the receiver-operator (ROC) curve) based on signal detection literature for quantifying these terms.

Predictive Power and Goodness of Fit. Presumably all ecosystem scientists are familiar with the concept of goodness of fit. At least in the case of model predictions that come in the form of continuous variables, there are welldeveloped and intuitive statistical measures of the goodness of fit of a model to a dataset. These include consideration of the related concept of bias. Håkanson 
(Chapter 8) explores the concept of goodness of fit in considerable detail and presents the concept of "predictive power" when the goodness of fit of a model can be tested a number of times (i.e., in different systems or settings).

\section{Model Output}

We follow Harald Bugmann's lead in Chapter 21 and consider four characterizations of model output. When applied to statements of the future states of ecosystems, the four terms are generally interpreted to imply differing degrees of certainty.

Prediction. Merriam-Webster's on-line dictionary defines "prediction" as "foretell on the basis of observation, experience, or scientific reason." There are at least two distinctly different usages in ecosystem modeling. Modelers adhere to the common definition when they refer to temporal predictions from dynamic models (i.e., statements about the future state of an ecosystem based on model output). Modelers commonly depart from the standard usage when they refer to any qualitative or quantitative output of a model as a "prediction," regardless of whether the model is static or dynamic. For example, a regression model "predicts" the primary productivity of a lake (without explicit reference to time) as a function of phosphorus loading (Chapter 8).

As Bugmann points out in Chapter 21, the common definition is often interpreted by the public (and resource managers) to imply a high degree of certainty. Scientists don't necessarily make this assumption and instead rely on a variety of measurements of the goodness of fit of the model predictions to observed data (Chapter 8). Oreskes (Chapter 2) argues that the predictive ability of ecosystem models is fundamentally limited because ecosystems are not "closed" systems and because important physical and biological forcing functions are necessarily treated as externalities. This clearly imposes limitations on goodness of fit of temporal predictions.

Forecast. Merriam-Webster's on-line dictionary defines "forecast" as "to calculate or predict some future event or condition, usually as a result of study and analysis of available pertinent data; to indicate as likely to occur." In the lexicon of modeling, the critical distinction between a prediction of a future event and a forecast lies in the assessment of the likelihood of the occurrence of the event (Chapter 21). As Clark et al. (2001) define it, an ecosystem forecast is "[a prediction of the] state of ecosystems... with fully specified uncertainties." They do not completely resolve the question of what would constitute "full specification" of uncertainty.

Projection. Merriam-Webster's on-line dictionary defines "projection" as "an estimate of future possibilities based on a current trend." The common usage seems to imply less certainty than either a prediction or a forecast (Chapter 21). In technical usage, it would appear to be most appropriately applied to the results of the broad range of techniques for extrapolating to future ecosystem states from past data, based on a statistical model.

Scenario. Defined as "an account or synopsis of a possible course of action or events" (Merriam-Webster's on-line dictionary), the term "scenario" appears 
to be most commonly used by ecosystem modelers in the sense of an "if/then" statement referring to the hypothetical predictions of a model under a specified set of parameter values, initial conditions, and external forcing functions (Chapter 21). While "scenarios" may not contain any statement of the likelihood of an actual, future state of a real ecosystem, the use of quantitative models to explore the logical consequences of alternative scenarios (i.e., given the structure of the model and its associated assumptions) is a powerful motivation for modeling in both basic and applied science (Chapter 7).

\section{Use of Models in Ecosystem Management}

For every problem there is a model that is simple, clean and wrong.adapted from a quote by H.L. Mencken on solutions to societal problems

If all models are simplifications of nature (Chapter 2) and therefore never fully capture the range of behavior of real ecosystems (Chapter 10), how "wrong" can a model be and still be useful in a management context? Håkanson (Chapter 8) defines a set of quantitative indices of how "wrong" models are in the context of errors of prediction. More generally, Pielke argues strongly for better communication of the limitations of both our basic understanding and the inherent predictability of ecosystems (Chapter 7). As he points out, management decisions are, and always will be, made in the face of imperfect knowledge. Very little is served (and real damage can be done) by the failure of scientists to clearly communicate the nature and uncertainty of model predictions.

Models serve a number of purposes in ecosystem management other than prediction and forecasting. These include providing decision-support systems for focusing consideration of diverse issues and providing an explicit framework for adaptive management (Chapter 16). As Harris et al. point out in Chapter 16 , the use of models in ecosystem management is now ubiquitous. It can be argued that the most innovative work on the development of ecosystem modeling as a tool in science is being done in the context of resource management.

\section{References}

Burnham, K.P., and D.R. Anderson. 1998. Model Selection and Inference: A Practical Information-Theoretic Approach. New York: Springer-Verlag.

Clark, J.S., S.R. Carpenter, M. Barber, S. Collins, A. Dobson, J.A. Foley, D.M. Lodge, M. Pascual, R. Pielke Jr., W. Pizer, C. Pringle, W.V. Reid, K.A. Rose, O. Sala, W.H. Schlesinger, D.H. Wall, and D. Wear. 2001. Ecological forecasts: An emerging imperative. Science 293: 657-660.

Fennel, K., M. Losch, J. Schroter, and M. Wenzel. 2001. Testing a marine ecosystem model: Sensitivity analysis and parameter optimization. Journal of Marine Systems 28: 45-63. 
Gardner, R.H., R.V. O’Neill, J.B. Mankin, and J.H. Carney. 1981. A comparison of sensitivity analysis and error analysis based on a stream ecosystem model. Ecological Modelling 12: 177-194.

Grace, J.B., and B.H. Pugesek. 1997. A structural equation model of plant species richness and its application to a coastal wetland. American Naturalist 149: 436-460.

Hilborn, R., and M. Mangel. 1997. The Ecological Detective: Confronting Models with Data. Princeton, NJ: Princeton University Press.

Oreskes, N., K. Shrader-Frechette, and K. Belitz. 1994. Verification, validation, and confirmation of numerical models in the earth sciences. Science 263: 641-646.

Starfield, A.M., K.A. Smith, and A.L. Bleloch. 1990. How to Model It: Problem Solving for the Computer Age. New York: McGraw-Hill. 


\section{Part I}

The Status and Role of Modeling in Ecosystem Science 
